\title{
Semitendinosus snapping: analysis of movement, electromyographic activities, muscle strength and endurance, motor control and joint position sense
}

\author{
Hande Guney ${ }^{1}$ \\ Defne Kaya ${ }^{2}$ \\ Caglar Yilgor 3 \\ Murat Cilli ${ }^{4}$ \\ Serdar Aritan 4 \\ Inci Yuksel ${ }^{1}$ \\ Mahmut Nedim Doral ${ }^{3}$
}

1 Physioterapy Department, Faculty of Health Sciences, Hacettepe University, Ankara, Turkey

2 Sports Medicine Department, Faculty of Medicine, Hacettepe University, Ankara, Turkey

3 Orthopaedics and Traumatology Department, Faculty of Medicine, Hacettepe University, Ankara, Turkey

${ }^{4}$ School of Sports Sciences and Technology, Hacettepe Univerity, Ankara, Turkey

Corresponding author:

Hande Guney

Physioterapy Department

Faculty of Health Sciences, Hacettepe University

06100, Sinhiye

Ankara, Turkey

E-mail: handeguney@hotmail.com

\section{Summary}

A female ballet with a history of two-years of semitendinosus (ST) snapping was assessed. On physical examination snapping was observed during hyperextension of the knee. Neither any history of trauma nor treatment was recalled. Magnetic resonance imaging (MRI), movement analysis, onset timing of ST and Bisceps Femoris (BF), motor control, isokinetic muscle strength and endurance, joint position sense (JPS) were assessed. The MRI findings were normal. There were abnormal oscillations observed during hyperextension of the snapping knee compared to healthy side. There were no isokinetic muscle strength nor do muscle endurance differences. The motor control and JPS deficits were greater on the snapping knee than the healthy side. ST onset timing was earlier than BF on the snapping side. Snapping of the semitendinosus tendon has an adverse affect on JPS, motor control and onset timing of the knee muscles.

KEY WORDS: snapping knee syndrome, motor control, movement analysis, joint position sense, ballet dancer.

\section{Introduction}

Snapping syndrome is defined as a feeling of tendon snapping or popping while performing an activity and mostly seen in the athletes ${ }^{1-7}$. Although snapping knee syndrome is a rare seen condition, this can lead to disabling problems ${ }^{1-4,6,8}$. In most cases symptoms involve lateral aspect of the knee and the underlying causes are associated with the presence of discoid meniscus ${ }^{9}$ or lateral meniscus tear ${ }^{7}$, biceps femoris ${ }^{4}$, intra-articular tumours ${ }^{10}$, proximal tibio-fibular joint instability ${ }^{11}$, and juxta-articular ganglion cysts ${ }^{12}$. Snapping around the medial aspect of the knee are even more uncommon ${ }^{1,3,5,13}$. Bollen et al. ${ }^{2}$ reported 4 cases of snapping in the medial knee caused by pes anserinus; they named this condition snapping pes syndrome.

To our knowledge, analysis of movement during snapping, isokinetic muscle strength, joint position sense and motor control in patients with semitendinosus snapping have not been investigated. Although there are studies which evaluate electromyographic activities and gait analyses in patients with medial snapping, the parameters included no details $s^{1,3,5}$. Therefore, the aim of this study is to analyze movement during snapping and investigate how snapping tendon effects muscle strength and endurance, electromyographic activities, joint position sense (JPS) and motor control.

\section{Method}

A 19-year-old female ballet presented with a history of two-years of semitendinosus snapping. Neither any history of trauma nor treatment was recalled. She recalled no pain during daily activities. The snapping occurred with hyperextension of the knee in neutral, internal and externally rotated positions. The patient experienced snapping only while dancing, especially during activities involving hyperextension of the knee such as fondue, battement frappe and adagio which are done as transition movements. Although she did not have pain, the snapping caused delayed harmony during dancing, and caused lack of synchronization with other dancers, thus caused stress; eventually forcing her to consider abandoning her career and discontinuing all strenuous activities. Patient was informed about aims of the study, and the testing procedure prior to her participation. Written informed consent was obtained. 
Semitendinosus snapping: analysis of movement, electromyographic activities, muscle strength and endurance, motor control and joint position sense

\section{Clinical Assessment}

The clinical examination included joint range of motions of the knee and hip, tightness of hamstrings, gastrocnemius, tensor fascia latae and quadriceps muscles were carefully noted. The clinical $Q$ angle and limb length discrepancy were measured. The subject herself acts as her own internal control by using the healthy side.

\section{Imaging}

Anteroposterior and lateral plain radiographs were obtained and magnetic resonance imaging (MRI) was performed on a 1.5 T Philips $^{\circledR}$ (Intera Achieva, Philips Medical Systems, The Netherlands) scanner. A body coil was used with the patient in the supine position. Axial T1-weighted turbo spin echo images (TR range/TE range, 375/7) of both knees were obtained.

\section{Movement Analyses}

Three Mini-DV cameras (Sony DCR-HC19) operating at 25 frame per-second (50 field per-second) were used to record the movement. Shutter speed was set to $1 / 500$. While the optical axis of two cameras was approximately placed to an angle of $45^{\circ}$, the third camera was placed vertical to the frontal plane of the subject. Firewire (IEEE 1394b) outputs of cameras were utilised for capturing and recording the video directly from the camera to the hard-disk drive on the computer. A matlab script was written to synchronize the video frames by using Data Acquisition Toolbox. A calibration frame with 8 control points was used for three-dimensional (3D) space (Fig. 1A). Kinematics of lover body of the subject was obtained from the points that were selected on the leg. Five anthropo- metric points were selected, which were toe, heel, ankle, knee and hip. There are also six additional markers were used to identify six-degree of freedom (6 DoF) of the movement of upper and lower legs (Fig. 1B). The fondue movement which causes snapping was repeated for 15 seconds in both extremities and records were obtained for three consecutive repeats $^{14}$.

The points were digitised using the HUBAG 3D Movement Analysis Software, then digitised data was transformed to real distance units using the calibration scale placed in the field of vision ${ }^{15}$. Transformation was achieved by utilising Direct Linear Algorithm (DLT). A second order low-pass digital filter "Butterworth", which is cut-off frequency $6 \mathrm{~Hz}$ was applied to the raw data. As a result erroneousness high frequency component of the displacement data were removed ${ }^{14}$.

The global positions of leg and thigh on a rigid segment are used in conjunction with anatomical calibrations to determine the body-fixed axes. Triads of markers placed on the lateral side of the both limbs. These external markers let determine Local Coordinate System (LCS) that allow us to calculate orientation of the upper and the lower legs. Thus two pieces of rigid plates were placed into subject's leg and thigh. Finally, the rotations of the limbs were calculated respect to the Global Reference Frame ${ }^{14}$.

\section{EMG Recording- Onset timing determination}

Electromyographic signals were obtained with selfadhesive silver/silver chloride, $4 \mathrm{~mm}$ radius disc surface electrodes. Electromyography of the biceps femoris (BF) and semitendinosus (ST) muscles was performed using an oscilloscope program with two channels in free run [Keypoint equipment (Medtronic, Copenhagen)]. Pairs of electrodes were positioned

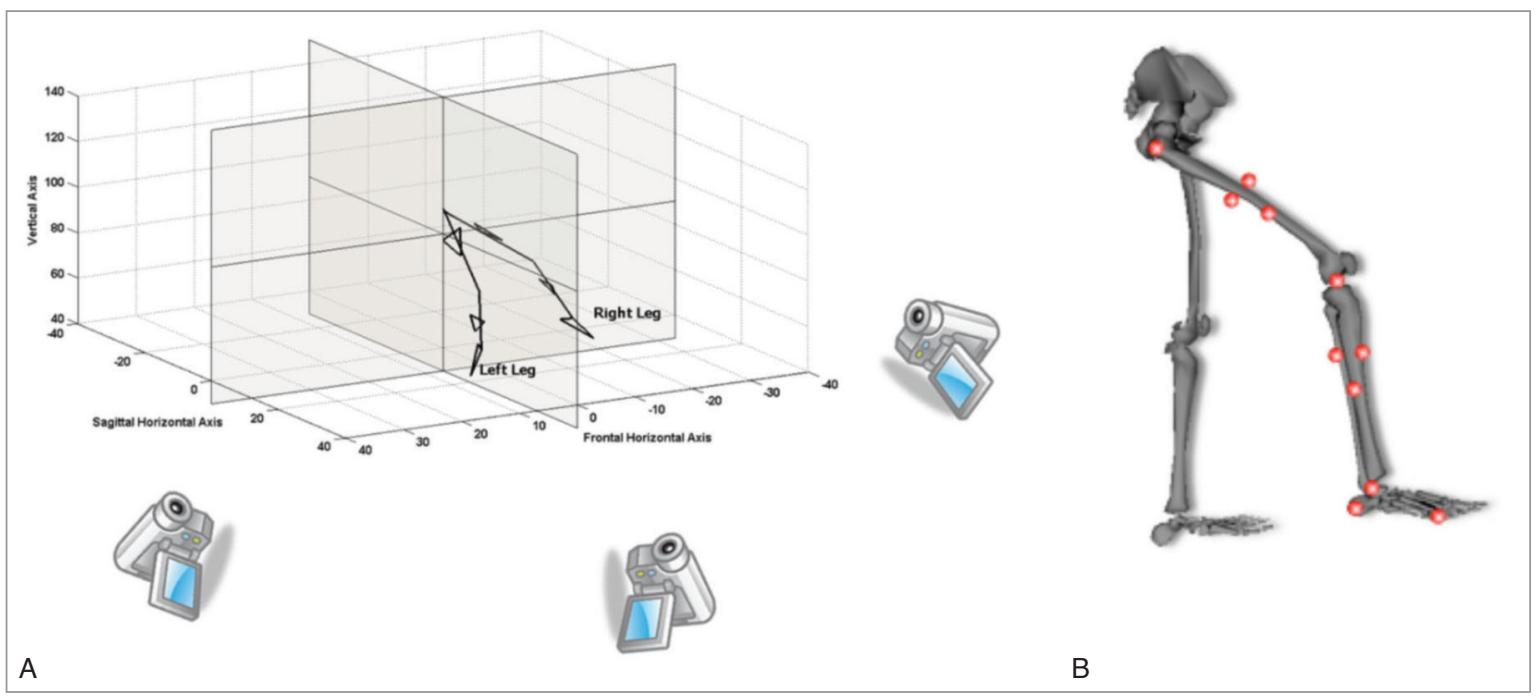

Figure 1. A) Camera positions and calibrated volume. B) Marker positions of 5 anthropometric points and two triad markers of upper and lower legs. 
with an inter-electrode distance of $1.5 \mathrm{~cm}$ each over the belly of the BF and ST muscles; halfway along the ischial tuberosity and the lateral epicondyle for the BF and halfway along the ischial tuberosity and the medial epicondyle for the ST ${ }^{15}$. The electrode locations were identified during a maximal knee isometric effort from the prone position. The grounding electrode was attached on the tibial tuberosity. The electrical impedance was reduced below $5 \mathrm{KW}$ by shaving and cleaning the skin with alcohol. The sweep speed was $160 \mathrm{~ms}$ and sensitivity was $0.2 \mathrm{~m} \mathrm{~V}$ per division. The amplifier bandwidth was preset from 5$10.000 \mathrm{~Hz}$ in each channel at sampled at $1000 \mathrm{~Hz}^{16}$.

Patient was informed to relax completely until a flat electrical baseline was seen on EMG channels to avoid movement artefact and noise before each trial. Then the patient performed a maximal volunteer isometric contraction of the knee flexors for $5 \mathrm{~s}$ after a verbal command. Tests were repeated in three different positions (limb was in neutral, in internal rotation and external rotation). The first deflection from the baseline was accepted as the onset of EMG activity and the onsets were marked. The relative difference in the time of onset of EMG activity of BF and ST was quantified during the task by subtracting the onset of ST from that of BF. A negative or low value therefore indicates that ST onset is before that of BF. EMG onsets and the relative difference were identified from trials and averaged over the three repetitions. The resting period between each isometric contraction was two minutes ${ }^{16}$.

\section{Motor control}

The multi-joint lower limb tracking-trajectory test as performed on a Functional Squat System (Monitored Rehab Systems, Haarlem, The Netherlands) was found reliable to assess motor control during concentric and eccentric joint movement (ICC values of 0.77 $0.80)^{17}$. The patient was placed in a single-leg halfsquat position in supine on this device with the hip, knee, and ankle joints flexed at $90^{\circ}$. For each patient, $20 \%$ of body weight as determined during relaxed stance on a platform scale was calculated and applied as the resistance load during the entire test. The concentric component of the test involved hip, knee, and ankle extension from a half-squat position to a position of complete knee extension by means of composite concentric activation of the lower limb extensor muscles. The eccentric component of the test involved returning to the half-squat position from full knee extension by means of eccentric activation of lower limb extensors with agonistic flexor muscle coactivation. After a standardized warm-up including 10 concentric-eccentric repetitions, subjects were allowed a 30-second practice trial prior to coordination test performance. The coordination test was started with the non-involved lower extremity with a $20 \%$ bodyweight load. The Functional Squat System device was interfaced with a computer that had a dedicated software program (Monitored Rehab System,
Haarlem, The Netherlands). This software converted angular knee joint movement to linear movement of a cursor on a video monitor so that patient had realtime visual feedback of their position during test performance. As horizontal squat depth increased with increasing knee flexion, the cursor moved to the left representing the eccentric movement phase. As horizontal squat depth decreased with increasing knee extension, the cursor moved to the right representing the concentric movement phase. Patient was instructed to direct the cursor along the pathway depicted on the video screen. Sixty-seconds of target tracking was completed, and the tracking error was calculated by device software provided real-time data analysis during both the eccentric and the concentric phase of the coordinative test.

\section{Muscle endurance}

The patient was placed in the same single-leg halfsquat position in supine on a horizontal squat machine ${ }^{17}$. The functional endurance test was completed unilaterally and consisted of 20 repetitions for each extremity. Resistance was $20 \%$ of body weight. Device software determined the squat force for each test repetition and the total muscle work following the completion of 20 test repetitions.

\section{Muscle strength}

Patient was evaluated using the ISOMED 2000 isokinetic dynamometer (D\&R Ferstl GmbH, Hemau, Germany $)^{18}$. She was seated with the knees and legs flexed $90^{\circ}$ to determine the isokinetic torque value of the hamstring and quadriceps during knee flexion and extension. The centre of the knee joint was aligned with the centre of the dynamometer using a laserpointing device. After a 5 minute warm-up, the angular velocity was set at $60 \%$ s. The patient was asked to push up the lever arm of the system 5 times as strongly as possible and return to the starting position. The same procedure was repeated at $180 \%$ with 10 repetitions after a break of one minute. The average maximum torque value was calculated. Differences in 'peak torque' of the snapping and healthy knee was calculated as a percentage.

\section{Joint position sense}

Joint position sense was measured by active reproduction test in the Functional Squat System. Gattie et al. ${ }^{19}$ showed that Functional Squat System ${ }^{\circledR}$ is a valid tool assessing joint proprioception in the clinical setting. Subjects were positioned in supine with the test knee flexed $90^{\circ}$ while the opposite foot was resting on device. A load of $20 \%$ bodyweight as previously determined was applied during test performance. As they viewed the device monitor, patient was instructed to keep the cursor on a defined pathway which provided 
Semitendinosus snapping: analysis of movement, electromyographic activities, muscle strength and endurance, motor control and joint position sense

her with continual knee position feedback. Following this, the patient was instructed to return to the start position of $90^{\circ}$ knee flexion and attempt to replicate the reference knee position without visual feedback of the cursor. The difference in linear cursor position between the reference and reproduction trial was calculated by device software. This value represented error during active joint angle reproduction testing.

\section{Results}

Clinical assessments: there were no difference in $Q$ angle, hip and ankle range of motions and range of knee flexions between the snapping knee and healthy knee. There was no tightness in hamstring, quadriceps, tensor fascia lata and gastrosoleus muscles on both lower legs. No leg length discrepancy was noted.
Imaging: there was no difference on plain radiographs when comparing both legs. Radio graphical $Q$ angle, Insall-Salvati index and posterior slope of the tibia were similar for both legs. The MRI of the snapping knee revealed no intra-articular pathology, meniscal tear or medial parapatellar plica. There were no signs of ligament rupture, fat pad pathology and bursitis. Thickness of the semitendinosus and the gracilis tendons were normal.

Movement Analyses: the time period for the snapping knee to reach full extension was $82 \%$ longer then the healthy side, conversely the time between full extension and the hyperextension was 18\% shorter (Figs. 2 and 3 ).

When observed in all three dimensions healthy side's motion is smooth, whereas the snapping knee movement involve involuntary angular oscillations. The biggest angular alternations were observed in the

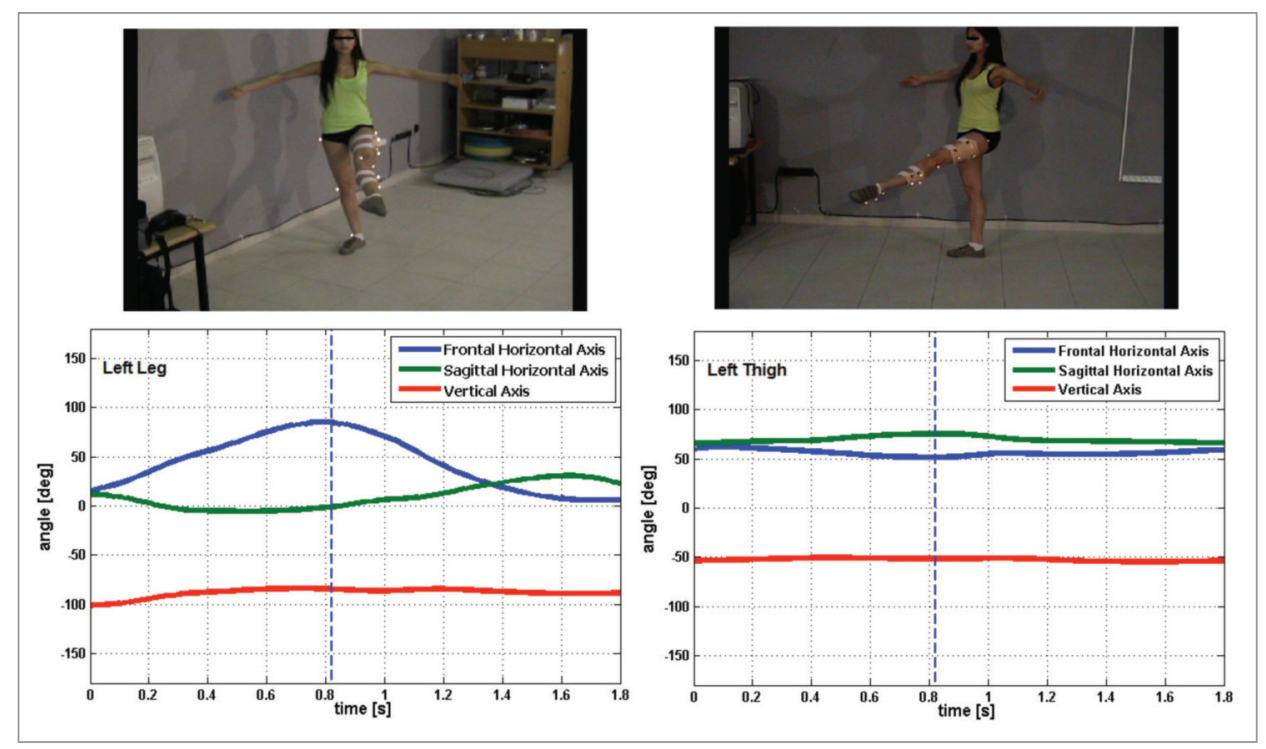

Figure 2. Angular rotation of the healthy knee and thigh with respect to the anatomical reference planes. Pictures from the video sequence show the moment of full extension of the healthy side.

Figure 3. Angular rotation of the snapping knee and thigh with respect to the anatomical reference planes. Pictures from the video sequence show the moment of full extension of the snapping side.

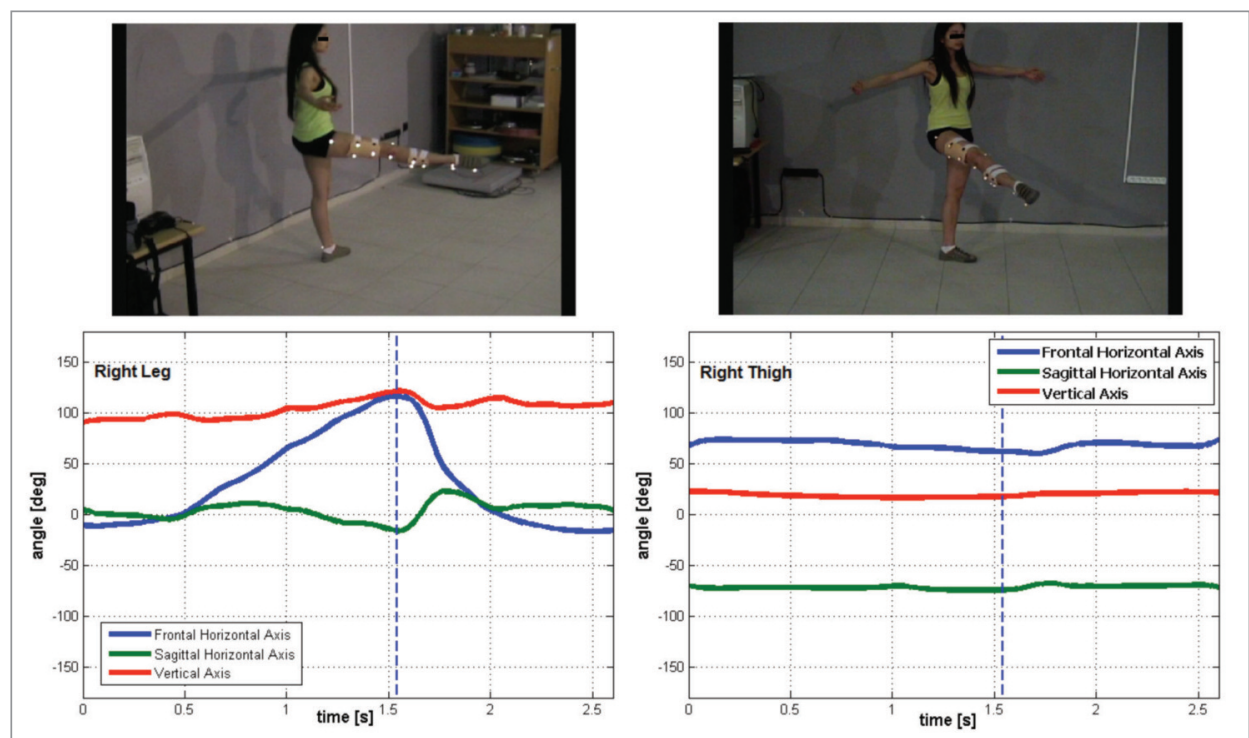


Table 1. Onset timing of Biceps and Semitendinosus on knee internal rotation, external rotation and neutral positions.

\begin{tabular}{lllll}
\hline Onset timing $(\mathbf{m s})$ & Position of the knee & Muscle & Snapping Knee & Healthy Knee \\
\hline & IR & ST & 485 & 886 \\
& BF & 465 & 889 \\
\hline ER & ST & 515 & 892 \\
& BF & 482 & 884 \\
\hline & Neutral & ST & 615 & 1090 \\
& BF & 572 & 1083 \\
\hline
\end{tabular}

IR: internal rotation, ER: external rotation, ST: semitendinosus, BF: biceps femoris

Table 2. Results in muscle endurance, muscle strength, motor control and joint position sense on both snapping and healthy side.

\begin{tabular}{|c|c|c|c|c|}
\hline \multicolumn{3}{|l|}{ Variables } & \multirow{3}{*}{$\begin{array}{l}\text { Snapping Knee } \\
489 \\
376\end{array}$} & \multirow{3}{*}{$\begin{array}{l}\text { Healthy Knee } \\
502 \\
396\end{array}$} \\
\hline Muscle Eno & rance $(\mathrm{Nm})$ & Concentric & & \\
\hline & & Eccentric & & \\
\hline \multirow{4}{*}{ 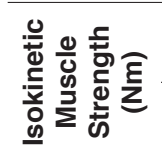 } & Quadriceps & $60 \% / s$ & 96 & 97 \\
\hline & & $180 \% / s$ & 79 & 82 \\
\hline & Hamstring & $60 \%$ & 64 & 60 \\
\hline & & $180 \% \mathrm{~s}$ & 78 & 66 \\
\hline \multirow{2}{*}{\multicolumn{2}{|c|}{$\begin{array}{l}\text { Motor control tracking } \\
\text { trajectory error }(\mathrm{cm})\end{array}$}} & Concentric & 2.8 & 1.1 \\
\hline & & Eccentric & 3.4 & 1.3 \\
\hline \multicolumn{3}{|c|}{ JPS reproduction error (cm) } & 4.5 & 1.8 \\
\hline
\end{tabular}

JPS: joint position sense

sagittal-horizontal axis between full extension and hyperextension, which corresponds to the snapping.

EMG Data: for the healthy knee the differences in onset timing between the BF and ST were $3 \mathrm{~ms}, 2 \mathrm{~ms}$ and $7 \mathrm{~ms}$ for IR, ER, and neutral positions of the knee, respectively. There was no difference in the onset timing between ST and BF on healthy side (Table 1).

For the snapping side, the differences in onset timing between the BF and ST were $20 \mathrm{~ms}, 33 \mathrm{~ms}, 43 \mathrm{~ms}$ for IR, ER, and neutral positions of the knee, respectively. There was an onset timing difference between ST and BF and ST was contracting earlier then BF (Table 1).

Motor Control: trajectory-tracking error was 2.6 times higher on snapping side than the healthy side on both eccentric direction and concentric direction (Table 2). Muscle Strength: similar peak torque values were recorded at $60 \%$ s and $180 \%$ s for both hamstring and quadriceps muscles (Table 2).

Muscle Endurance: functional endurance levels did not differ between the snapping side and healthy side (Table 2).

Joint Position Sense: the deficit of joint position testing was 2.5 times higher on snapping side than the healthy side (Table 2).

\section{Discussion}

The principal finding was the snapping knee represented a greater deficit in joint position sense and motor control. Additionally there was an imbalance in onset timing of ST and BF muscles.
Previous studies mostly focused on the surgical treatment and the causes mostly specify on the anatomic variances of the tendons and the bones $2,3,5,6,13,20,21$. Lyu and $\mathrm{Wu}^{5}$, explained the mechanism of semitendinosus snapping by dissecting ten knees of five cadavers without snapping symptoms and pointed out the "fanned out fibers". They indicated that during knee extension and tibia's internal rotation, fanned out fibers become tense and lead the tendon slips from the groove. They also added that hyperextension with internal rotation force the tibial condyle to rotate posteriorly and increase the tendency of semitendinosus tendon to slip out ${ }^{5}$. In another study, the mechanism of the semitendinosus snapping in an athlete was explained as the repetitive vertical jumping with the knee in hyperextension position causes excessive loading and forces the tendons to displace $^{3}$. In our study, during movement analysis, the biggest oscillations were seen between full extension and hyperextension on the snapping knee comparing to the healthy side. This suggests that hyperextension mechanism forced the tendon to snap. Also, this finding supports the previous findings on hyperextension mechanism has a negative influence on the semitendinosus tendon.

Previous studies defined possible anatomical causes but it was still unknown that how the snapping tendons affect the JPS, motor control and onset timing of the muscles. In our patient, JPS deficit (2.5 times) and motor control deficit (2.6 times) on snapping side were found higher than the healthy side. It is well known that there is a close relationship between sensory system function and motor control. Functional move- 
Semitendinosus snapping: analysis of movement, electromyographic activities, muscle strength and endurance, motor control and joint position sense

ments depend on acquisition of stimuli from peripheral mechanoreceptors in joints, muscles, tendons and deep tissues ${ }^{22,23}$. They transfer information to the central nervous system about the duration, direction, amplitude, speed, acceleration/deceleration, and timing of movement ${ }^{24}$. The central nervous system, via projection of these stimuli, regulates motion with agonistantagonist muscle activity $22,25,26$. Tracking accuracy relies on merging sensory activity and position sense information in the cerebral cortex $22,24,26$. Multi-modal sensory information enables the central nervous system to establish an internal model or central representation during motor learning. This internal model is related to joint position, velocity of movement, and force output information $22,24,26$. The results of our study indicate that snapping tendon causes a neuromotor regulation system impairment. Additionally, differences in onset timing of the ST relative to the BF was a proposed mechanism for snapping tendon and was related to this motor regulation system impairment. No differences were observed for muscle strength and endurance, but we believe that abnormal JPS, motor control and EMG findings might influence these parameters negatively in future.

In the literature, it's been well established that surgical intervention is the best treatment for the snapping symptoms especially for pain 1-7,20,21. Our patient neither have pain, nor wanted to have surgery. Since, our results showed the adverse effect of snapping semitendinosus tendon on neuromotor system, we believe that surgical intervention is needed.

\section{Conclusions}

This study showed that snapping of the semitendinosus tendon influence the joint position sense, motor control and onset timing of the knee muscles adversely. We did not perform any surgery, but we recommend a detailed assessment and an appropriate surgical intervention especially for the athletes who have snapping knee syndrome to prevent future problems.

\section{Acknowledgement}

The authors wish to thank Ahmet Ozgur Atay and Cagri Temucin who provided valuable assistance to the assessments and Gul Baltaci who guide us on using the research laboratory. Authors declare that they have no sponsor in the study design, in the collection, analysis and interpretation of data; in writing of the manuscript; and in the decision to submit the manuscript for publication.

\section{Conflict of interest statement}

All authors have no conflicts of interest with respect to the data collected and procedures used within this study.

\section{Ethical statement}

The authors confirm this study meets the guidelines of the Declaration of Helsinki. Patient was informed about aims of the study, and the testing procedure prior to her participation. Written informed consent was obtained.

\section{References}

1. Bae DK, Kwon OS. Snapping knee caused by the gracilis and semitendinosus tendon. A case report. Bull Hosp Jt Dis 1997; 56(3):177-179.

2. Bollen SR, Arvinte D. Snapping pes syndrome: a report of four cases. J Bone Joint Surg Br 2008; 90(3):334-335.

3. Karataglis D, Papadopoulos P, Fotiadou A, Christodoulou AG. Snapping knee syndrome in an athlete caused by the semitendinosus and gracilis tendons. A case report. Knee 2008; 15(2):151-154.

4. Lokiec F, Velkes S, Schindler A, Pritsch M. The snapping biceps femoris syndrome. Clin Orthop Relat Res 1992; (283):205-206.

5. Lyu SR, Wu JJ. Snapping syndrome caused by the semitendinosus tendon. A case report. J Bone Joint Surg Am 1989; 71(2):303-305.

6. Cooper DE. Snapping popliteus tendon syndrome. A cause of mechanical knee popping in athletes. Am J Sports Med 1999; 27(5):671-674.

7. Park JH, Ro KH, Lee DH. Snapping knee caused by a popliteomeniscal fascicle tear of the lateral meniscus in a professional Taekwondo athlete. Orthopedics 2012 July 1; 35(7):e1104-e1107.

8. Malliaropoulos NG. Non contact Hamstring injuries in sports. Muscles Ligaments Tendons J 2013; 2(4):309-311.

9. Kocher MS, Klingele K, Rassman SO. Meniscal disorders: normal, discoid, and cysts. Orthop Clin North Am 2003; 34(3):329-340.

10. Mine $T$, Ihara $K$, Taguchi $T$, et al. Snapping knee caused by intra-articular tumors. Arthroscopy 2003; 19(3):E21.

11. Nadaud MC, Ewing JW. Proximal tibiofibular arthritis: an unusual cause of lateral knee pain. Orthopedics 2001; 24(4):397-398.

12. Liu PC, Chen $\mathrm{CH}$, Huang $\mathrm{HT}$, et al. Snapping knee symptoms caused by an intra-articular ganglion cyst. Knee 2007; 14(2):167-168.

13. Geeslin AG, LaPrade RF. Surgical treatment of snapping medial hamstring tendons. Knee Surg Sports Traumatol Arthrosc 2010; 18(9):1294-1296.

14. Abdel-Aziz YI, Karara HM. Direct linear transformation from comparator coordinates into object space coordinates in close-range photogrammetry. Proceedings of the Symposium on Close-Range Photogrammetry pp. 1-18. Falls Church, VA: American Society of Photogrammetry 1971.

15. Aritan S, Cilli M, Amca AM. HUBAG: Three Dimensional Movement Analysis Software. Hacettepe J of Sport Sciences 2010; 21(1):30-36.

16. Lynn SK, Costigan PA. Changes in the medial-lateral hamstring activation ratio with foot rotation during lower limb exercise. J Electromyogr Kinesiol 2009 ;19(3):e197-e205.

17. Maffiuletti NA, Bizzini M, Schatt S, Munzinger U. A multi-joint lower-limb tracking-trajectory test for the assessment of motor coordination. Neurosci Lett 2005 12; 384(1-2):106-111.

18. Dirnberger J, Wiesinger HP, Stoggl T, Kosters A, Muller E. [Absolute and relative strength-endurance of the knee flexor and extensor muscles: a reliability study using the IsoMed 2000-dynamometer]. Sportverletz Sportschaden 2012; 26(3):142-147. 


\section{H. Guney et al.}

19. Gattie ER, Decoster LC, Heon MM, LaRoche DP. Reliability and validity of the monitored rehabilitation functional squat proprioception test component. http://www.nhmi.net/validity_and_reliability_of_the_monitored_rehab.php

20. Bernhardson AS, LaPrade RF. Snapping biceps femoris tendon treated with an anatomic repair. Knee Surg Sports Traumatol Arthrosc 2010; 18(8):1110-1112.

21. Date H, Hayakawa K, Nakagawa K, Yamada H. Snapping knee due to the biceps femoris tendon treated with repositioning of the anomalous tibial insertion. Knee Surg Sports Traumatol Arthrosc 2012; 20(8):1581-1583.

22. Solomonow M, Krogsgaard M. Sensorimotor control of knee stability: a review. Scand J Med Sci Sports 2001;11:64-80.

23. Dhillon MS, Bali K, Prabhakar S. Differences among mechanoreceptors in healthy and injured anterior cruciate ligaments and their clinical importance. Muscles Ligaments Tendons J 2012 Jun 17; 2(1):38-43.

24. Lephart SM, Pincivero DM, Rozzi SL. Proprioception of the ankle and knee. Sport Med 1998; 25:149-155.

25. Zhang Q, Joshi SK, Manzano G, Lovett DH, Kim HT,Liu $X$. Muscle extracellular matrix degradation and contractibility following tendon rupture and disuse. MLTJ 2013; 3(1): 35-41.

26. Yosmaoglu HB, Kaya D, Guney H, Nyland J, Baltaci G, Yuksel I, Doral MN. Is there a relationship between tracking ability, joint position sense, and functional level in patellofemoral pain syndrome? Knee Surg Sports Traumatol Arthrosc 2013 Jan 30. 